\title{
sciforum
}

\section{Peptide therapy reinforced with nanotechnology: an innovative strategy for controlled drug delivery}

\author{
Aline Edurne San Juan Meza
}

${ }^{a}$ Universidad de Guadalajara, División de estudios de la Biodiversidad,, Departamento de Ciencias

de la Tierra y la Vida

Minireview

\begin{tabular}{|l|l|} 
Graphical Abstract & $\begin{array}{l}\text { Abstract. } \\
\text { Recently, nanosciences have opened a large number of } \\
\text { possibilities to find timely treatments for multiple diseases; } \\
\text { Cancer or Alzheimer's are being addressed with therapies } \\
\text { based on focusing the treatment in a highly selective way } \\
\text { that guarantees greater efficacy in the dose and prolonged } \\
\text { effect. } \\
\text { The peptide systems are designed from the specific } \\
\text { characteristics of a pathology, they have an adequate } \\
\text { volume to send many units to the target with high specificity. } \\
\text { However, it is a duel to deliver them to the target, since in } \\
\text { the course they are likely to be anabolized or accumulate in } \\
\text { the surrounding tissue. } \\
\text { This is the reason why nanomaterials are an alternative to } \\
\text { create systems that offer them the resistance to reach their } \\
\text { recognition cells. The most studied transporters include } \\
\text { nanoparticles, hydrogels, microemulsions, liposomes, } \\
\text { micelles and polymers. } \\
\text { Below, its advantages and applications in systems coupled to } \\
\text { peptides with therapeutic activity are described. }\end{array}$ \\
\hline
\end{tabular}

\section{1._INTRODUCCION}

Peptide systems are designed from specific markers of a certain pathology, use unique chemical signals that allow them to reduce the possibility of side effects by focusing their field of action.

They also have a molecular volume small enough to be produced in large quantities, while they are as advanced than high molecular weight molecules. Despite these characteristics, they are still sensitive to metabolism, to physiological changes and may accumulate outside the target.; Together, these factors reduce the half-life of the treatment and limit its application.

Therefore, biocompatible vehicles are needed that protect the therapeutic load and guarantee its delivery in the pharmacological receptor. For the production of these systems should be considered the route of administration, the half-life of the dose and the process of its biodegradation, and this is where nanomaterials can be exploited, since they would be created with the chemical versatility to resist the route and can also be functionalized in accordance with the requirements for the target.[1] 


\section{2._PEPTIDES TECHNOLOGY}

The peptides act as pharmacological targets thanks to the changes adopted and the existence of specific sequences of recognition towards them. When a complex is successful, it manages to cross the bilayer and can act as an inhibitor of interactions, ligand or catalyst.

This is used, for example, in the case of insulin as the treatment for diabetes. Its peptide is composed of 51 amino acids in two chains linked by disulfide bridges and is supplied for the activation of the GLUT4 carriers for the mobilization of glucose through interaction with its respective receptor.

We also have the case of Ziconotide a peptide extracted from the paralytic toxin of the aquatic snail that works as an analgesic in cases of chronic pain, using the selective blockade of the N-type calcium channels, which is applied instead of opioids such as morphine.

Other peptides are designed to inhibit infection processes or prevent the viral interaction that triggers the fusion and replication of these agents. In fact, most antibiotics are derived from peptides; Its production uses recombinant microorganisms, transgenic models, or chemical synthesis, the latter being a strategy with many obstacles, but with a wide range of amino acid combinations.

However, the therapies have the disadvantage that few molecules are internalized and still them are susceptible to a lysosomal degradation, so higher and continuous doses will be needed to maintain the therapeutic effect.

Other types of peptides that do not have therapeutic activity are used as pharmacological spikes to cross the biological barriers, the peptides that penetrate the CPP cells are short sequences positively charged, amphipathic and abundant in lysine or arginine with the ability to transfer the load to the cell and cross the bilayer. One recently studied is the TAT peptide derived from HIV type 1, whose penetration capacity is used to boost the shipments of nanocarriers coupled to drugs.

Whether due to its pharmaceutical activity or as a barrier overstep tool, the study of peptides must be improved, and alternatives are presented below to counteract the main problem, its delivery.

\section{3._NANOCARRIERS COUPLED TO PEPTIDES}

\section{1._Microemulsions}

They are monophasic mixtures where oil and water coexist, which with the help of surfactants generate stable thermodynamic systems[1], the proportion of their components determines their nature, there may be systems water in oil or vice versa, this depends on the polarity of the drug that wants to emulsify[2], in addition should be consider the distribution of the charge present in the surfactant because it will be the main responsible for stabilizing the system.

Thanks to their easy preparation and stability they are able to protect fragile drugs, especially peptide, can solubilize lipophilic drugs and improve their bioavailability. [3] o / w microemulsions have been used to solubilize steroids and hormones but only offer advantages if the drug is highly soluble in the oil phase. [4] w / o systems are reported for intramuscular release of a luteizing hormone analogue to regulate the testosterone levels in rats, as well as for the delivery of cliclosporin, insulin and other peptides. There is even talk of the production of multiple systems $\mathrm{w} / \mathrm{o} / \mathrm{w}$ for this purpose [5].

Also, to optimize absorption and dissolution of active principles, isotropic mixtures have been developed that form nanometric drops that prevent the dissolution in the intestine of hydrophobic peptide drugs such as vasopressin.[6] 


\section{2._Solid- Lipid Nanoparticles}

They are spherical particles constructed from a lipid core and a surfactant coating as a stabilizing agent [5], differ from emulsions in that they are solid at body temperature and their administration can be topical, oral or intravenous.

The prolonged release of this type of systems is achieved thanks to the gradual action of the lipid matrices whose internal structure houses the drug in question, which can have any type of polar nature, these structures are constructed through physiological lipids, therefore they are completely biocompatible, you can choose different types of emulsifiers for their synthesis although generally the number is reduced to those that can be used for parental administration[9].

Its main application is for the administration of insulin by means of a nebulizer although a treatment with modified particles is also reported that has achieved high absorption of the peptide in the intestine. Hence the importance of functionalizing its surface to extend its application. Among its disadvantages are: the variability in size due to its growth, its possible gelling[1], and its obstinacy to incorporate drugs due to its crystalline structure.

\section{3._Liposomes}

They are structures based on lipids that are formed in a colloidal dispersion, depending their number of bilayers can be unilamellar or multilamellar, dissolve hydrophobic drugs in their membranes while hydrophilic or amphipathic compounds are maintained in their aqueous center[4]. To supply bioactive molecules, they fuse with the cellular bilayer and delivery substances that is not able to penetrate the membrane by itself. However, this method can deform them; such is the case of the mellitin that is used in the treatment of HIV that when interacting with the membrane induces the formation of pores and the leakage of the drug.

The size and charge of the liposome can be manipulated by conjugating them with polymers such as PEG, this modification increases their circulation time and achieves their accumulation in tumor sites[1]. However, the coating with PEG is not easily degraded, therefore, hybrid PEG-peptide coatings have been proposed which, upon contact with the acidic surface of the vacuole during endocytosis, break the liposome and release the drug.

Another area of interest is the development of immunoliposomes that achieve selective vectorization by incorporating antibodies to their bilayer. So that the antigens of the tumor are used to fix to the liposome and later release the pharmacological load.

\section{4._Polymeric Micelles}

Its structure is made up of self-assembled amphiphilic polymers, it has a hydrophobic core that solubilizes fat-soluble drugs and a hydrophilic external crown that contacts the medium, this isolated arrangement between the drug and the exterior increases the physicochemical stability of this. They are physically stable against dilution, and remain aggregated even after dilution below a critical point.

Its structure is designed to adjust the length of the blocks and their hydrophilic-lipophilic ratio to achieve special characteristics of porosity or micro viscosity and have a sustained supply of the drug at an adequate speed. Its surface can be modified with special ligands that can be captured by specific cells.

Taking advantage of the micellar disposition peptide-based therapies have been designed to treat ovarian cancer. According to one study, the micelles are conjugated with peptides OA02[1], this ligand targets the alpha 3 receptors of the integrin and the treatment attack the vasculature of the tumor. Finally, the 
properties of the drug, such as the molecular weight, the partition coefficient, $\log \mathrm{P}$, the tendency to aggregation and the presence of specific functional groups also condition it is incorporation into the micelle.

\section{5._Hydrogel}

They are crosslinked polymers that absorb large amounts of water without dissolving and form elastic structures[16]. For drug delivery systems, they use the hydration and relaxation of the polymer chain that spreads the drug when the gel is tumescent, the repetition of this operation speeds up the process.[1]

For its synthesis, polymers sensitive to small physicochemical changes that generate important responses in properties or phases are sought. This sensitivity is regulated by classical interactions, such as hydrogen bridges or ionic forces, these interactions mediate the hydrogel's drying-swollen transition.

The most promising breakthrough with hydrogels occurred when the release of insulin in the intestine was achieved, in this trial it is reported that the insulin was protected from acid hydrolysis[9] with chitosanbased hydrogel that at the right moment mobilizes the degradation of the vehicle by the proteases and the release of the bioactive, after which the glucose levels in the rats studied were reduced by $40 \%$.[1]

\section{4._CONCLUSIONS}

The research in controlled release techniques has given some important results for the storage and directed delivery of peptides, the studies show that the conjugated systems evolve towards a personalized design that allows to increase the bioavailability of the drug and evade the hydrolytic response or the loss of bioactivity due to changes in the environment.

The various degrees of specialization with which their surfaces are covered have allowed a concrete delivery of the substance to be achieved in most cases. It is understood that the main advantage offered by these systems is their ability to interact with cell membranes whether they merge with them or cross them. However, the stability problems that these vehicles have and the inherent disturbances that arise in the environment, warn us that although these technologies are already an applicable reality, there is a design gap that should be refined in the coming years.

\section{5._REFERENCES}

1._ Narayansaswamy, R.,Wang T., Torchilin V.P.Improving Peptide Appliations Using Nanotechnology,Current Topics in Medical Chemistry,Rev,2016,16(1), 280-253.

2._Carlucci, A., Cicconi, M, Bregni C. Las Microemulsiones como vehículos para la administración de Drogas. Acta Farm. Bonaerense 2004, 23 (4): 550-7.

3._Trotta, M., M. Gasco \& F. Pattarino Acta Pharm. Technol. 1990, 36: 226-31.

4._ Du, AW; Stenzel M.H. Drug carriers for the delivery of therapeutic peptides. Biomacromulecules, 2014,15(4),1097-114.

5._Morozowich, W. y Gao, P. Improving the oral absorption of poorly soluble drugs using SEDDS and S-SEDDS formulations. Elsevier Inc. 2009, 468-443.

6 Mehnert, W; Mader, K. Solid lipid nanoparticles: production characterization and applications. Adv. Drugs Deliv. Rev.,2001, 47(2-3), 165-96.

7._Jores, K., Mehnert W., Bunjes H., Drechsler M., and Mäder K. From solid lipid nanoparticles (SLN) to nanospoons. Visions and reality of colloidal lipid dispersions. In: $30^{\text {th }}$ INTERNATIONAL Symposium on Controlled Release of Bioactivity Material, pp1-2. 
8 Lim,S.B,:Banerjee, A,: Onyuksel, H. Improvement of drug safety by the use of lipid-based nanocarriers, J. Control Release, 2012, 163(1), 45-34.

9 RATNER, Buddy D; HOFFMAN, Alan S; SCHOEN, Frederick J; LEMONS, Jackie E. Biomaterials Science. An Introduction to Materials in Medicine. Third Edition. Elsevier. 2013. ISBN 978-0-12374626 